\title{
Gastrointestinal Bleeding Successfully Treated Using Interventional Radiology
}

\author{
Nobuhiro Takeuchi ${ }^{\mathrm{a}, ~ c}$, Masakazu Emori ${ }^{\mathrm{a}}$, Makoto Yoshitani ${ }^{\mathrm{a}}$, Junichi Soneda ${ }^{\mathrm{a}}$, \\ Masanori Takada ${ }^{\text {, }}$ Yusuke Nomura ${ }^{b}$
}

\begin{abstract}
Gastrointestinal (GI) bleeding is an emergency medical condition that leads to hemorrhagic shock or circulatory instability if left untreated. A mainstay for treating GI bleeding is endoscopic therapy; more than $90 \%$ of GI bleeding can be staunched by endoscopic hemostasis. However, patients with unstable hemodynamics or GI bleeding that cannot be controlled by endoscopy require transcatheter embolization or surgical intervention. The development of several devices and embolization agents that are used in interventional radiology (IVR) leads to safe and accessible treatment via IVR. If endoscopic treatment fails, IVR is the second strategy. Herein, we report cases of GI bleeding that were successfully treated by IVR and discuss the therapeutic strategy.
\end{abstract}

Keywords: Gastrointestinal bleeding; Interventional radiology

\section{Introduction}

Gastrointestinal bleeding (GI) is a critical condition that requires emergency hemostasis. It is widely recognized that emergency endoscopic hemostasis can be chosen as a first option for treating upper GI bleeding. However, endoscopic hemostasis may not be adequate for patients with circulatory instability or high risks. If circulatory conditions are unstable, or GI bleeding cannot be staunched or controlled by an endoscopy, interventional radiology (IVR) or surgical intervention is considered. Moreover, GI bleeding is characterized by the nature of intermittent bleeding. Extravasation is apparent when there is active bleeding; namely, responsible vessels cannot be detected unless there is evidence of bleeding. In such a case, empiric therapy, preventative embolization of possible culprit vessels, or provocative strategies for revealing extravasation,

Manuscript submitted May 10, 2017, accepted May 31, 2017

${ }^{a}$ Department of International Medicine, Kobe Tokushukai Hospital, Kobe, Japan

${ }^{b}$ Department of Internal Medicine, Kawasaki Hospital, Kobe, Japan

${ }^{\mathrm{c} C}$ Corresponding Author: Nobuhiro Takeuchi, Department of Internal Medicine, Kobe Tokushukai Hospital, 1-3-10 Kamitakamaru, Tarumi-Ku, Kobeshi, Hyogo 655-0017, Japan. Email: nobuhiro.takeuchi@tokushukai.jp

doi: https://doi.org/10.14740/gr851e including superselective catheterization or use of vasodilators should be considered. Surgical intervention is an option when both endoscopic hemostasis and IVR fail; however, IVR can be used proactively and repeatedly due to its feasibility and safety. Herein, we present cases of patients with GI that were successfully treated using IVR.

\section{Case Reports}

\section{Case 1}

A 71-year-old male visited our hospital by ambulance after he vomited a large amount of blood. Upon arrival, he was alert, blood pressure was $90 / 61 \mathrm{~mm} \mathrm{Hg}$, and heart rate was $73 \mathrm{bpm}$. Complete blood count (CBC) revealed mild anemia: red blood count $(\mathrm{RBC})$ was $395 \times 10^{4} / \mu \mathrm{L}$ and hemoglobin level was 12.0 $\mathrm{g} / \mathrm{dL}$. Emergency upper gastrointestinal (UGI) endoscopy revealed massive coagulated blood over the stomach, with no visual field secured (Fig. 1a). Active bleeding from the stomach was discovered. RBC dropped to $274 \times 10^{4} / \mu \mathrm{L}$ and hemoglobin level dropped to $8.1 \mathrm{~g} / \mathrm{dL}$; subsequently, administration of 6 units of red cell concentration (RCC) was required. Endoscopic hemostasis was abandoned and transcatheter arterial embolization (TAE) was planned. Before angiography, contrast-enhanced computed tomography (CT) was performed, and the results suggested extravasation from the posterior walls of the lower stomach body (Fig. 1b). Celiac angiography revealed extravasation from the posterior gastric artery (Fig. 1c). A microcatheter was advanced into the branch of the posterior gastric artery (Fig. 1d) and it was occluded by a gelatin sponge (Fig. 1e). On the following day, UGI endoscopy revealed successful hemostasis and Bormann 3 type cancer at the posterior walls of the lower gastric body (Fig. 1f). Biopsies taken from the gastric cancer revealed moderately-differentiated adenocarcinoma. His hemoglobin levels were elevated to $10.0 \mathrm{~g} /$ dL. However, on day 25, he fell into hemodynamic instability because of re-bleeding from exposed vessels of the cancerous lesion. Therefore, emergency surgery was performed with total gastrectomy. The postoperative course was uneventful.

\section{Case 2}

A 66-year-old male presented with blood in stools and dizzi- 

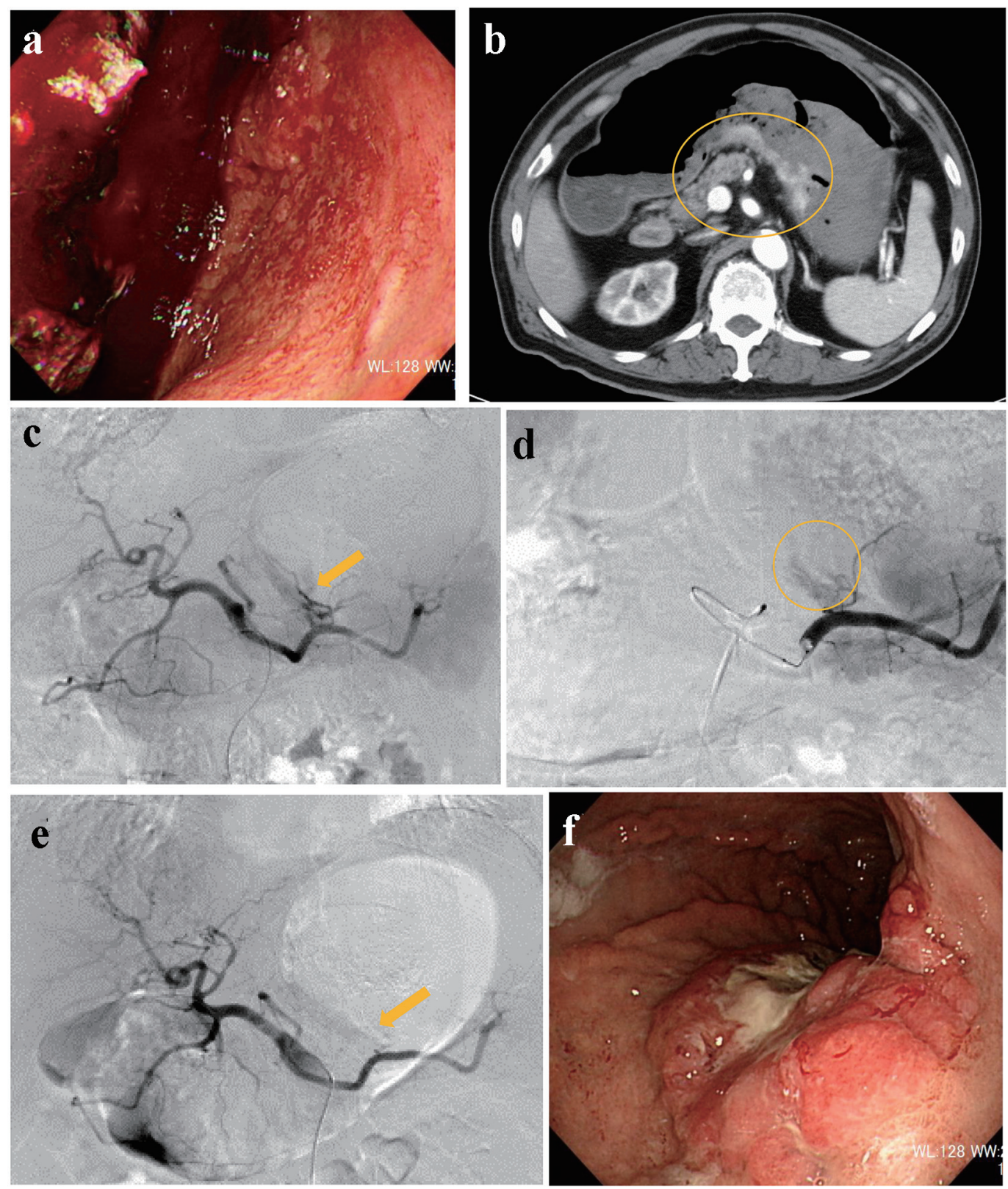

Figure 1. (a) Endoscopy reveals massive coagulated clots over the stomach. (b) Contrast-enhanced computed tomography suggests extravasation from the posterior walls of the lower stomach body (circle). (c) Angiography reveals extravasation from the posterior gastric artery (arrow). (d) A microcatheter is engaged into the branch of the posterior gastric artery (circle). (e) The responsible artery is successfully occluded by a gelatin sponge (arrow). (f) Endoscopy reveals Bormann 3 type cancer at the posterior walls of the lower gastric body.

ness. His medical history included chronic hepatitis B, which was followed up regularly without medication. On arrival, he was drowsy, blood pressure was $131 / 61 \mathrm{~mm} \mathrm{Hg}$, and heart rate was $98 \mathrm{bpm}$. CBC revealed moderate anemia: RBC was $277 \times$ $10^{4} / \mu \mathrm{L}$ and hemoglobin level was $8.4 \mathrm{~g} / \mathrm{dL}$. Non-contrast CT suggested retention of coagulated clots in the stomach. Subsequently, he was referred for emergency UGI endoscopy. UGI endoscopy revealed moderately retained fresh bleeding, coagulated clots, and Bormann 2 type cancer with exposed vessels at the angle of the stomach (Fig. 2a). On examination, there was no active bleeding. The exposed vessels were staunched by endoscopic clips (Fig. 2b). Immediately after UGI treatment, his consciousness level dropped to Glasgow coma scale E1V1M1 with a systolic blood pressure of $60 \mathrm{~mm} \mathrm{Hg}$. CBC revealed severe anemia: $\mathrm{RBC}$ was $180 \times 10^{4} / \mu \mathrm{L}$ and hemoglobin level was $5.5 \mathrm{~g} / \mathrm{dL}$. Due to re-bleeding from the gastric cancer, he was referred for angiography. After left gastric artery (LGA) angiography, a microcatheter was advanced into the distal LGA, and the contrast injection revealed extravasation (Fig. 2c). The responsible vessel was occluded using micro- 

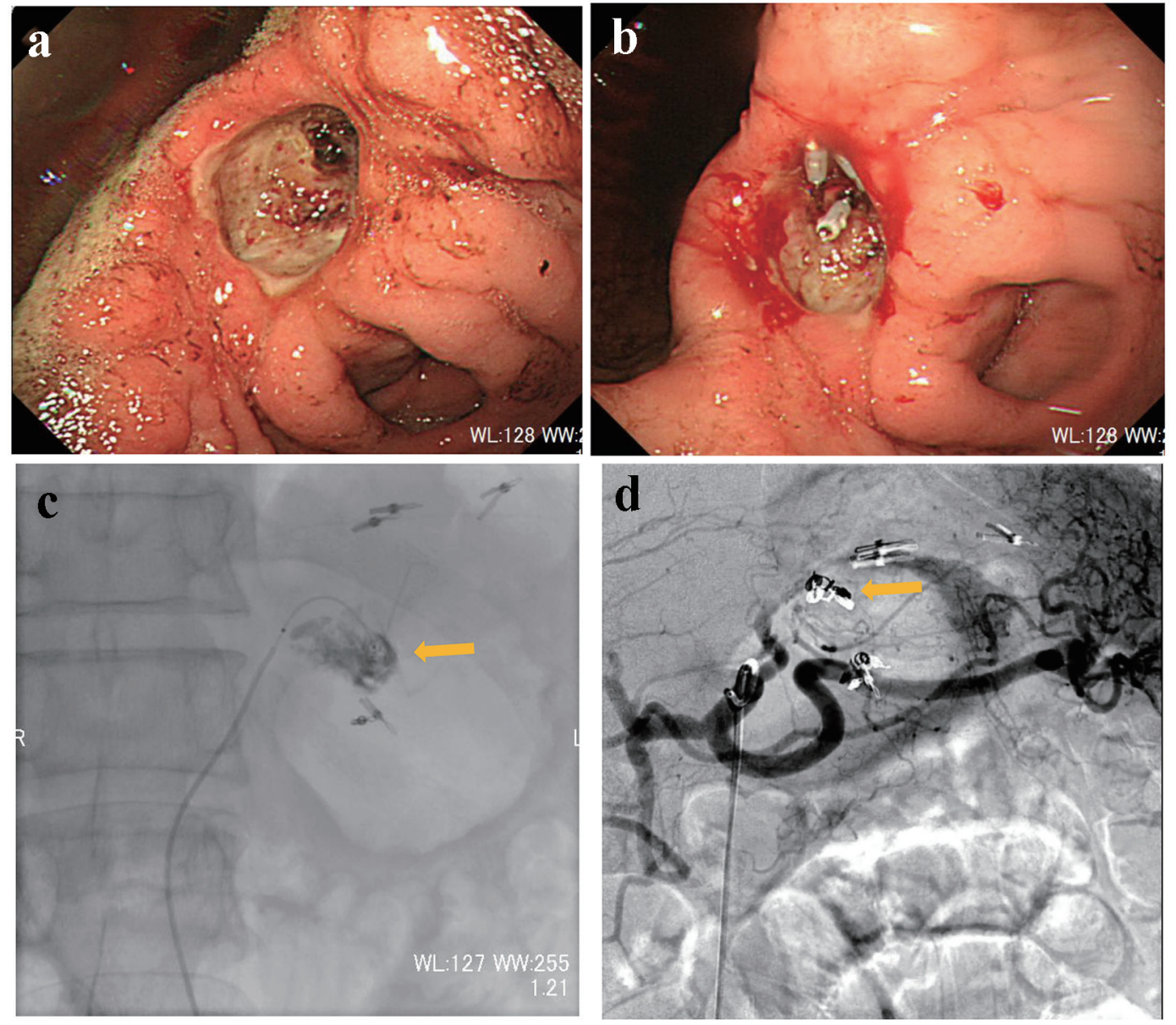

Figure 2. (a) Endoscopy reveals Bormann 2 type cancer with exposed vessels at the angle of the stomach. (b) The exposed vessels are staunched by endoscopic clips. (c) Contrast injection from a microcatheter advanced into the left gastric artery reveals extravasation (arrow). (d) The main trunk of the left gastric artery is occluded by microcoils (arrow).

coils (Fig. 2d). On day 3, UGI endoscopy confirmed successful hemostasis. Biopsies taken from the gastric cancer revealed poorly differentiated adenocarcinoma with signet-ring cells. On day 25, distal gastrectomy with Billroth-I reconstruction was performed. The postoperative course was uneventful.

\section{Case 3}

A 75-year-old male presented with melena. He could not walk unassisted; therefore, he visited our hospital by ambulance. His medical history included type 2 diabetes mellitus and benign prostatic hyperplasia, which were treated by oral medication. On arrival, his consciousness was unclear, blood pressure was $85 / 56 \mathrm{~mm} \mathrm{Hg}$, and heart rate was $104 \mathrm{bpm}$. CBC revealed severe anemia: RBC was $229 \times 10^{4} / \mu \mathrm{L}$ and hemoglobin level was $7.1 \mathrm{~g} / \mathrm{dL}$. After administration of 4 units of RCC, his hemoglobin level was elevated to $8.4 \mathrm{~g} / \mathrm{dL}$. On the following day, UGI endoscopy was performed, revealing coagulated clots in the anterior walls of the duodenal bulb. He was treated conservatively with fasting and prescription of proton pump inhibitor (PPI). On day 7, he presented with dizziness, he- matemesis, and melena. RBC and hemoglobin level dropped to $180 \times 10^{4} / \mu \mathrm{L}$ and $5.7 \mathrm{~g} / \mathrm{dL}$, respectively. UGI endoscopy revealed oozing at the duodenal bulb (Fig. 3a). Endoscopic hemostasis was considered challenging because of the unsecured visual field; therefore, he was referred for angiography. After gastroduodenal artery (GDA) angiography (Fig. 3b), a microcatheter was advanced into the distal right gastro-omental artery. Contrast injection revealed extravasation (Fig. 3c); then, the main trunk of the right gastro-omental artery was occluded by microcoils (Fig. 3d). On day 9, UGI endoscopy revealed successful homeostasis of the suspected exposed vessel (Fig. $3 \mathrm{e})$. Gastrography under endoscopy revealed the narrowed duodenal bulb (Fig. 3f). He did not clinically present the symptoms of duodenal obstruction. Serum pylori antibody was positive; then, eradication treatment of Helicobacter pylori was provided. Thereafter, the clinical course was uneventful.

\section{Case 4}

An 82-year-old male visited our hospital by ambulance, presenting with a low-grade fever, dyspnea, and melena. On ar- 

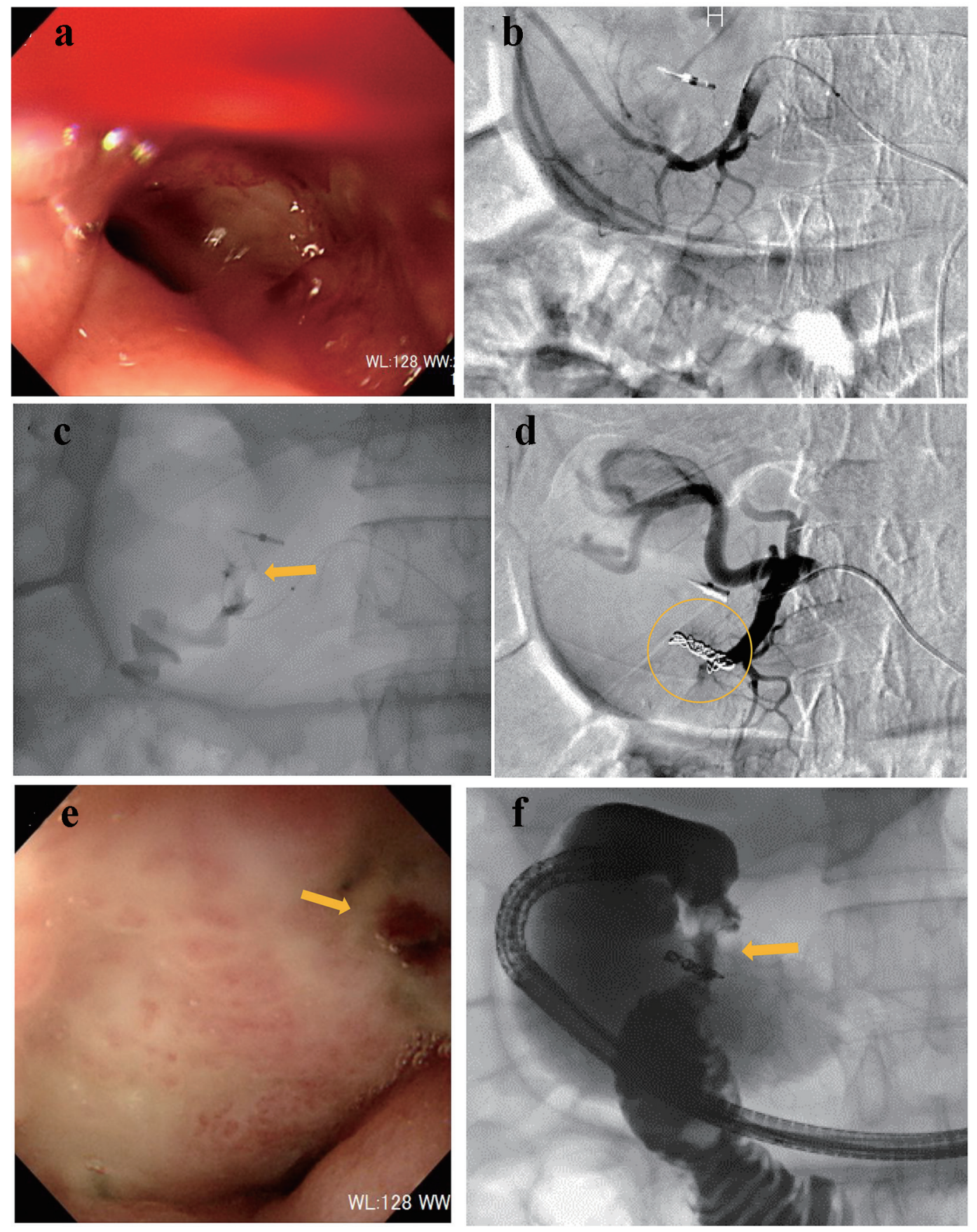

Figure 3. (a) Endoscopy reveals oozing at the duodenal bulb. (b) Gastroduodenal angiography reveals no extravasation. (c) Contrast injection from a microcatheter advanced into the right gastro-omental artery reveals extravasation (arrow). (d) The main trunk of the right gastro-omental artery is occluded by microcoils (circle). (e) Endoscopy confirms successful hemostasis (arrow). (f) Gastrography under endoscopy reveals the narrowed duodenal bulb (arrow).

rival, he was drowsy, blood pressure was $150 / 68 \mathrm{~mm} \mathrm{Hg}$, and heart rate was $79 \mathrm{bpm}$. His medical history included cerebral infarction. He was provided with enteral nutrition via a percutaneous endoscopic gastrostomy feeding tube. Laboratory analysis revealed severe anemia (RBC $199 \times 10^{4} / \mu \mathrm{L}$ and hemoglobin level $6.7 \mathrm{~g} / \mathrm{dL}$ ), abnormal renal function (creatinine $3.25 \mathrm{mg} / \mathrm{dL}$ and blood urea nitrogen $175.6 \mathrm{mg} / \mathrm{dL}$ ), and ab- normal electrolytes $\left(\mathrm{Na}^{+} 156 \mathrm{mEq} / \mathrm{L}, \mathrm{K}^{+} 7.4 \mathrm{mEq} / \mathrm{L}\right.$, and $\mathrm{Cl}^{-}$ $122 \mathrm{mEq} / \mathrm{L}$ ). Subsequently, transfusion and RCC were administered, and emergency hemodialysis for hyperkalemia was initiated. On day 3, UGI endoscopy was performed, revealing an exposed vessel at the posterior walls of the descending duodenum. An attempt to staunch the exposed vessel was made using endoscopic clips; however, oozing was uncontrol- 

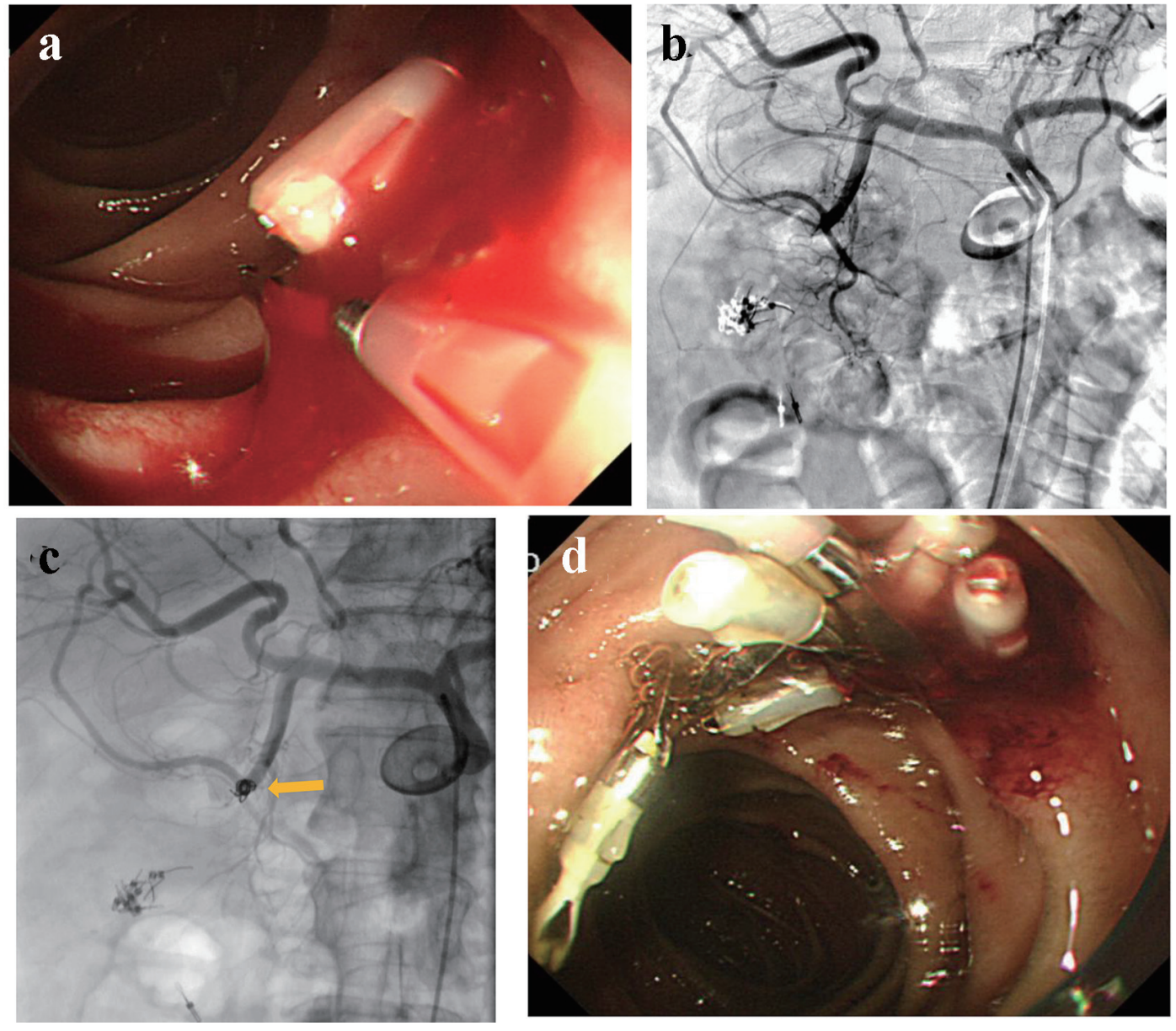

Figure 4. (a) An exposed vessel at the posterior walls of the descending duodenum is revealed and endoscopic hemostasis is attempted. (b) Gastroduodenal angiography reveals no evidence of extravasation. (c) Anterior superior pancreatico-duodenal artery is occluded by microcoils (arrow). (d) Endoscopy reveals successful hemostasis.

lable (Fig. 4a). Then, he was referred for angiography. GDA angiography revealed no evidence of extravasation (Fig. 4b); subsequently, empiric embolization was planned. With endoscopic clips, the anterior superior pancreatico-duodenal artery (ASPDA) was considered the responsible vessel. Therefore, the ASPDA was occluded using microcoils (Fig. 4c). UGI endoscopy on the same day revealed successful hemostasis (Fig. 4d). Administration of PPI was continued. Re-bleeding did not occur during his hospital stay.

\section{Case 5}

A 63-year-old male presented with blood in stools. His past medical history included coronary artery disease which was treated by coronary stenting and dual anti-platelet therapy, and hypertension and dyslipidemia that were treated by oral medicine. He also had a medical history of diverticular hemorrhage that was treated with endoscopic hemostasis. Upon arrival, he was alert, blood pressure was $158 / 74 \mathrm{~mm} \mathrm{Hg}$, and heart rate was $71 \mathrm{bpm}$ with regular rhythm. CBC revealed no anemia: $\mathrm{RBC}$ was $434 \times 10^{4} / \mu \mathrm{L}$ and hemoglobin level was $13.7 \mathrm{~g} / \mathrm{dL}$.
Emergency colonoscopy revealed multiple diverticulosis in the ascending colon with active bleeding (Fig. 5a). Endoscopic hemostasis failed; then, he was referred for angiography. Before angiography, contrast-enhanced CT was performed, revealing extravasation at the distal superior mesenteric artery (SMA) (Fig. 5b). After SMA angiography, a microcatheter was advanced into the distal SMA. Contrast injection from the microcatheter revealed extravasation (Fig. 5c). Distal SMA was occluded by a microcoil (Fig. 5d).

\section{Case 6}

An 82-year-old female presented with blood in stools. Her past medical history included coronary artery disease which was treated by coronary stenting and dual anti-platelet therapy, and atrial fibrillation that was treated by anti-coagulant medicine. On arrival, she was alert, blood pressure was 112/72 mm Hg, and heart rate was $71 \mathrm{bpm}$ with irregular rhythm. CBC revealed mild anemia: RBC was $400 \times 10^{4} / \mu \mathrm{L}$ and hemoglobin level was $10.9 \mathrm{~g} / \mathrm{dL}$. Emergency colonoscopy revealed multiple diverticulosis in the ascending colon without evidence 

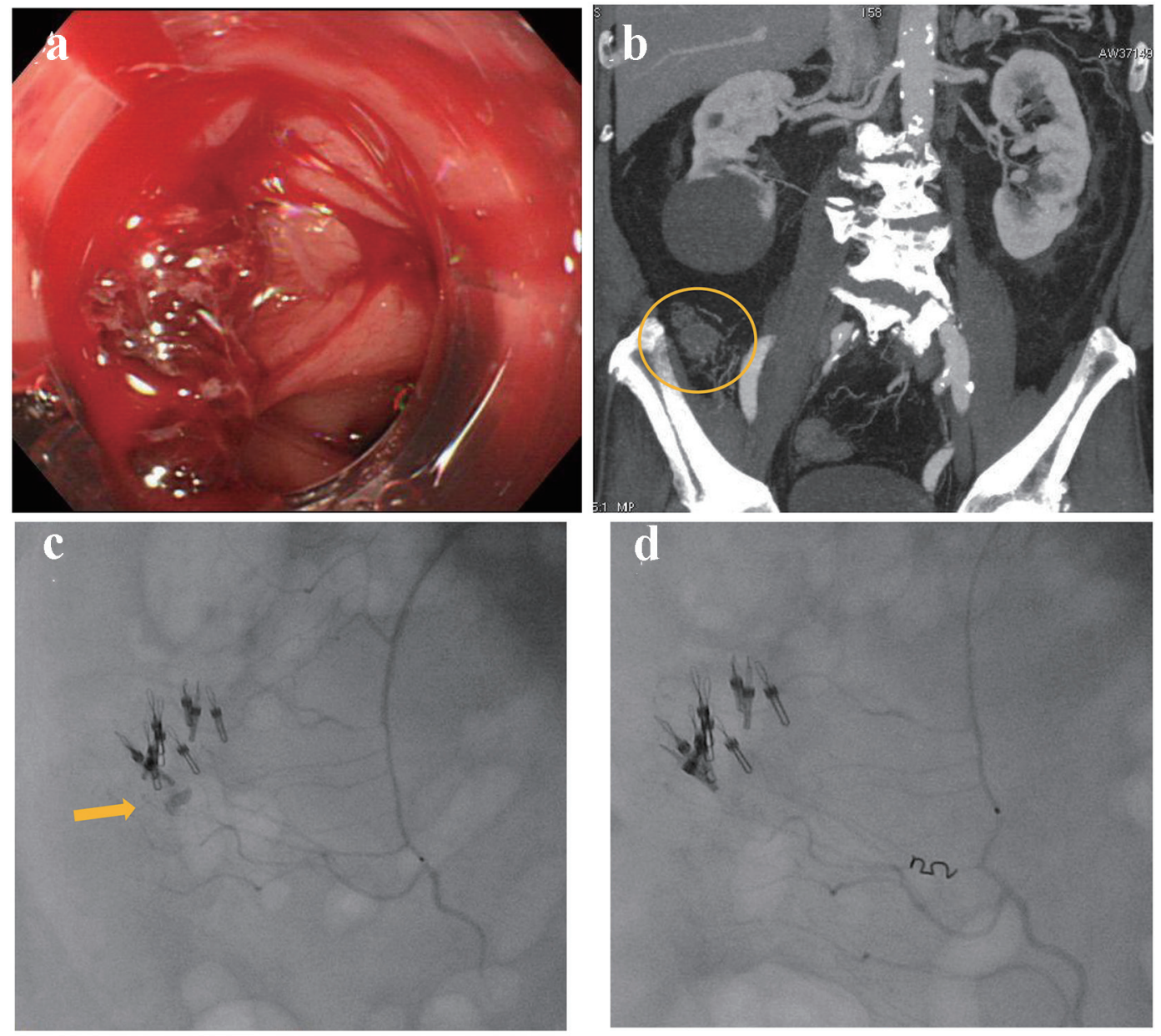

Figure 5. (a) Colonoscopy reveals multiple diverticulosis in the ascending colon with active bleeding. (b) Contrast-enhanced computed tomography reveals extravasation at the distal superior mesenteric artery (circle). (c) Contrast injection from a microcatheter reveals extravasation (arrow). (d) The distal superior mesenteric artery is occluded by a microcoil.

of bleeding (Fig. 6a). On the following day, 99m-labelled red blood cell scintigraphy was performed, suggesting the bleeding sites in the ascending colon (Fig. 6b). Subsequently, contrast-enhanced CT was performed, suggesting extravasation at the middle of ascending colon (Fig. 6c). Due to worsening anemia, administration of 6 units of RCC was required. The patient was referred for angiography. SMA angiography revealed extravasation from the middle colic artery (Fig. 6d). A microcatheter was advanced into the distal middle colic artery and it was occluded by a gelatin sponge. On the following day, colonoscopy revealed successful hemostasis and mild colonic ischemia (Fig. 6e). The colonic ischemia was observed by a conservative treatment.

\section{Case 7}

A 93-year-old female presented with blood in stools during her hospitalization due to acute cholecystitis. Her medical history included chronic renal failure and colon polyps that were resected by endoscopy 3 years ago. Emergency colonoscopy re- vealed ulcerative scars with a suspected exposed vessel at sigmoid colon $40 \mathrm{~cm}$ from anal canal, but there was no evidence of active bleeding (Fig. 7a). Therefore, she was observed by a conservative treatment. On the following day, she excreted $1,000 \mathrm{~g}$ of bloody stools and, subsequently, her blood pressure dropped to $74 / 50 \mathrm{~mm} \mathrm{Hg}$. Endoscopic hemostasis was considered challenging because of the possibly unsecured visual field; thereafter, he was referred for angiography. After the inferior mesenteric artery (IMA) angiography, a microcatheter was advanced into the distal IMA. Contrast injection revealed extravasation (Fig. 7b) and the distal IMA was occluded by a gelatin sponge (Fig. 7c). On day 4, colonoscopy revealed successful homeostasis with mild ischemic changes at sigmoid colon (Fig. 7d) that were observed with a conservative treatment.

\section{Discussion}

Endoscopic hemostasis is an established technique as a first choice for treating GI bleeding. Lower GI bleeding can be 

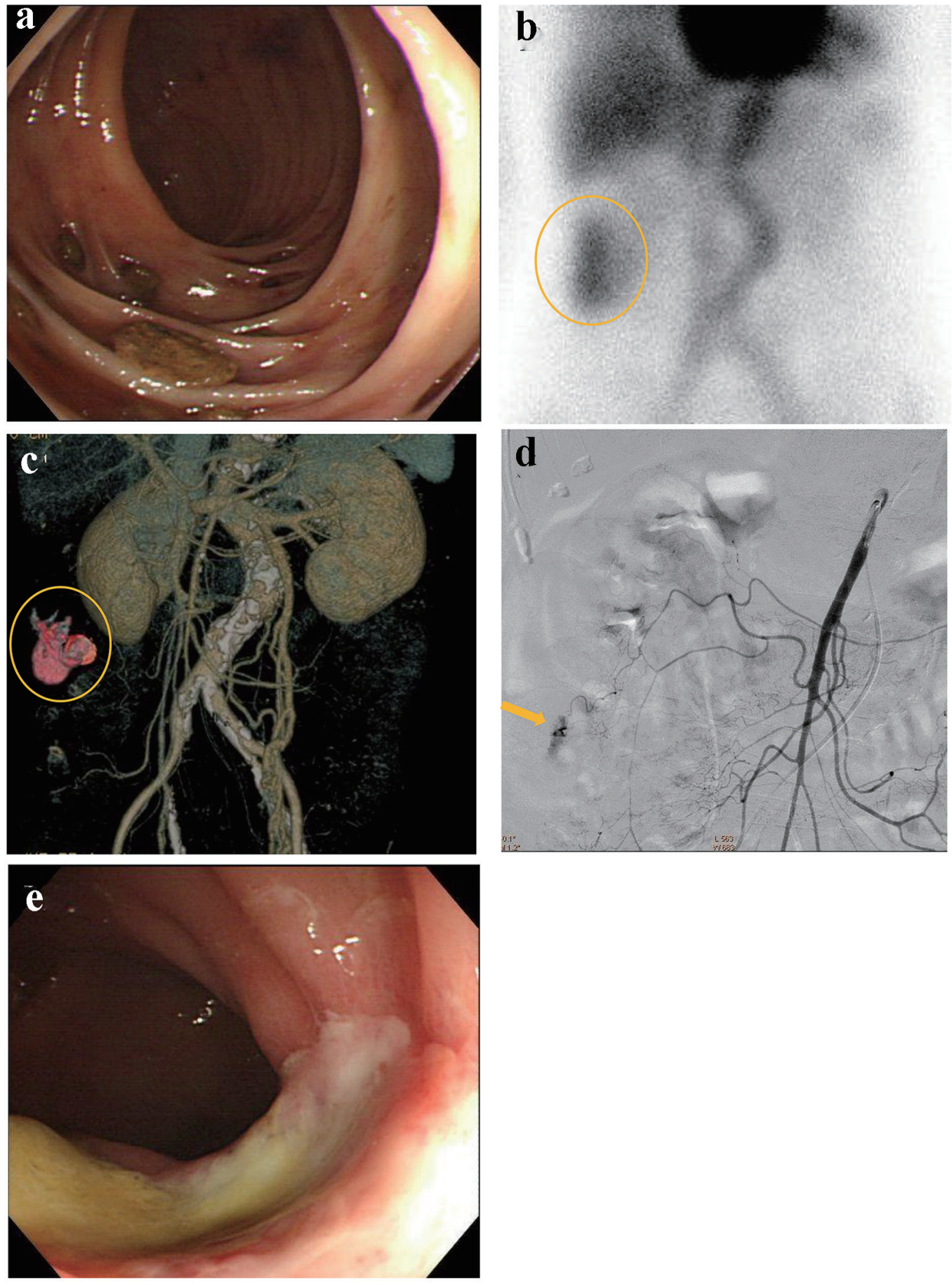

Figure 6. (a) Colonoscopy reveals multiple diverticulosis in the ascending colon without evidence of bleeding. (b) $99 \mathrm{~m}$-labelled red blood cell scintigraphy suggests the bleeding sites in the ascending colon (circle). (c) Contrast-enhanced computed tomography suggests extravasation at the middle of ascending colon (circle). (d) Superior mesenteric artery angiography reveals extravasation from the middle colic artery (arrow). (e) Colonoscopy reveals successful hemostasis and mild colonic ischemia.

observed conservatively, assuming the amount of bleeding is slight and the circulatory condition is stable; the majority of lower GI bleeding stops spontaneously. In contrast, upper GI bleeding should be addressed because continuous bleeding may be likely to cause hemorrhagic shock. The success rate of hemostasis under endoscopy is reported to be $90 \%$ [1]. Approximately half of acute GI bleeding is caused by gastric and duodenal ulcers [2]. Currently, the first option for treating GI bleeding is endoscopic hemostasis, including endoscopic clipping, injection of epinephrine or hypertonic saline-epinephrine 

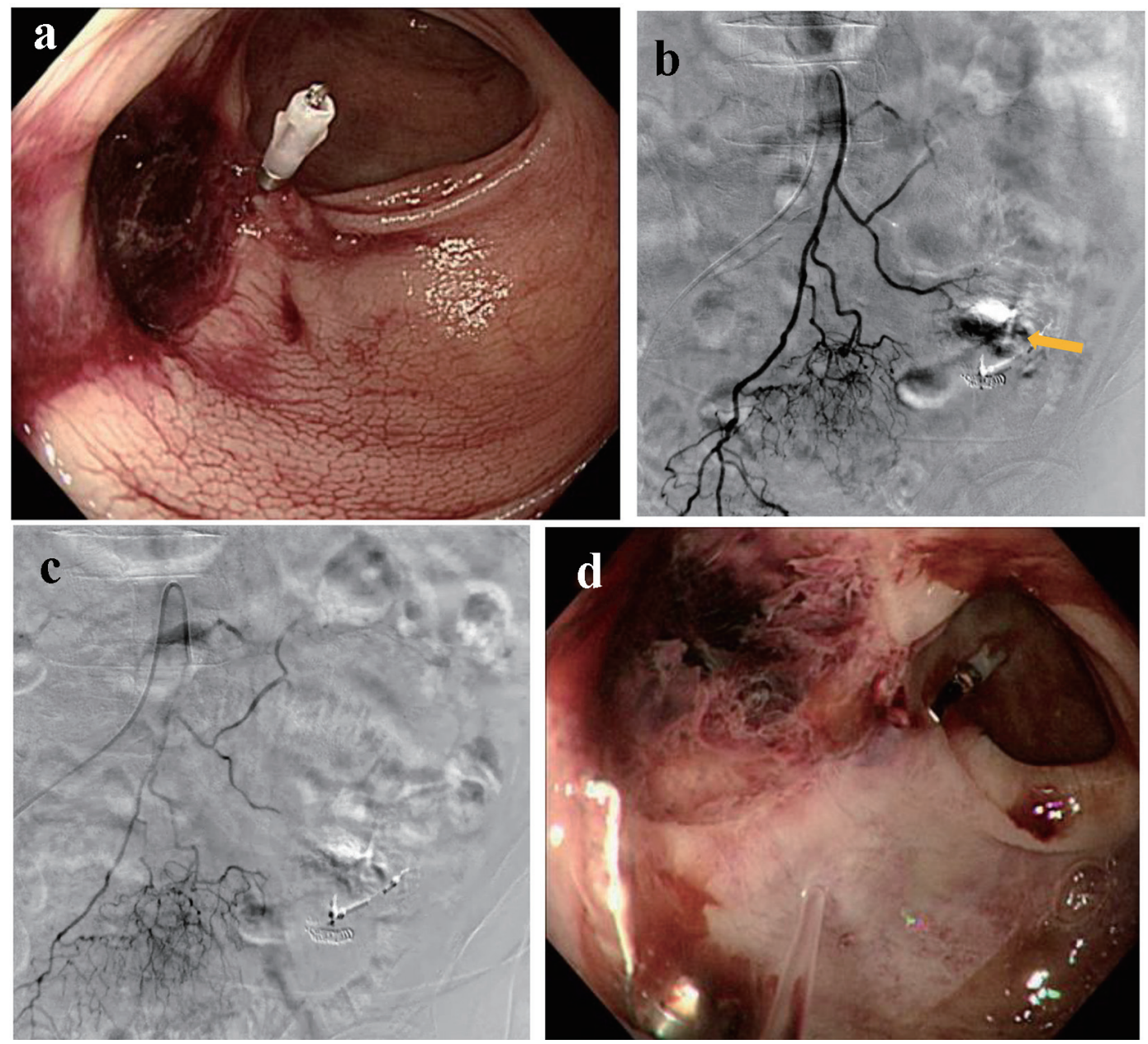

Figure 7. (a) Colonoscopy reveals ulcerative scars with a suspected exposed vessel at sigmoid colon $40 \mathrm{~cm}$ from anal canal without evidence of active bleeding. (b) Inferior mesenteric artery angiography reveals extravasation (arrow). (c) The distal inferior mesenteric artery is occluded by a gelatin sponge. (d) Colonoscopy reveals successful homeostasis with mild ischemic changes at sigmoid colon.

(HSE), and argon plasma coagulation. Endoscopic treatment with GI bleeding from peptic ulcers by epinephrine injection reportedly yields high rates of successful hemostasis ranging from $97.4 \%$ to $100 \%$ [3]. Clearly, endoscopic hemostasis is effective and feasible if circulatory conditions are stable and visual fields are secured. However, there are several cases where endoscopic hemostasis may fail in unfavorable conditions, including unstable hemodynamic status, respiratory failure, and poor visual fields. GI bleeding that is difficult to hemostasis under endoscopy or re-bleed may necessitate IVR or surgical intervention. It is reported that $10-30 \%$ of GI bleeding that is staunched by endoscopy may re-bleed $[4,5]$. Patients with massive GI bleeding may be at risk for deterioration of their general condition due to several postoperative complications; therefore, IVR is the optimal secondary option for treating GI bleeding.

Candidates for IVR include the following: 1) acute GI bleeding that is refractory to endoscopic management, 2) massive bleeding that requires transfusion of more than 4 units of blood over $24 \mathrm{~h}$, or unstable hemodynamic status or hemor- rhagic shock in which systolic blood pressure is $<100 \mathrm{~mm} \mathrm{Hg}$ and heart rate is $>100 \mathrm{bpm}$, and 3 ) recurrent bleeding.

In case 4, empirical embolization was adopted due to lack of angiographic evidence of bleeding. Because of the intermittent nature of GI bleeding, the detection of culprit vessels is sometimes challenging in angiographic examinations. The literature concerning patients with acute upper and lower GI bleeding reports that $52 \%$ of angiography reveals normal or negative results [6]. Frequency of angiographic negative results is significantly higher in patients with lower GI bleeding and stable hemodynamic conditions. In such cases, provocative angiographic investigations or empirical embolization are attempted. Provocative angiographic examination is a method to localize bleeding arteries with the use of intra-arterial tissue plasminogen activator, heparin, or trazoline [7, 8].

While empirical embolization is a method taken as a preventative measure for re-bleeding [9]. With the superselective catheterization of patients with GI bleeding, the percentage of positive angiography may increases to $93.4 \%$ [10]. However, it is noted that empirical embolization is controversial because 
of the increased possibility of re-bleeding and ischemia [9].

Transarterial embolization (TAE) in the upper GI tract above the Treiz ligament is unlikely to cause intestinal infarction because of the rich collateral arteries to the stomach and duodenum. However, with the wide range of TAE, rates of duodenal strictures may increase as seen in case 3 [11]. On the other hand, TAE in the lower GI tract may cause intestinal infarction unless TAE was performed in the vasa recta. This is because lower GI tract is characterized by poorer collateral vessels and anastomoses compared to upper GI tract.

Embolization agents are chiefly selected by the location of responsible vessels. Gelfoam, microcoils, N-butyl cyanoacrylate (NBCA), and the combination of these materials are commonly used when culprit vessels are occluded. In cases 1 , 6 , and 7, a gelatin sponge was used because the culprit vessels were superselected by a microcatheter. In other cases, microcoils were chosen for occluding the main trunk of LGA, GDA, and ASPDA, and the distal SMA with wider vessel diameter ranging from 2 to $4 \mathrm{~mm}$.

Re-bleeding after TAE sometimes occurs ranging from $21.0 \%$ to $34.4 \%$, possibly because of the development of collateral arteries $[12,13]$. Once GI bleeding is staunched by TAE, GI malignancies should be removed surgically as soon as possible or meticulous follow-ups by endoscopy should be performed (additional treatment should be considered if lesions are likely to re-bleed).

\section{Conclusion}

Herein, we reported seven cases of GI bleeding that were successfully treated using IVR. Clearly, endoscopic hemostasis is standard for GI bleeding, but IVR should be considered in GI bleeding patients with high risk, circulatory insufficiency, refractory bleeding, and poor visual fields. Although selective catheterization is preferable for detecting responsible vessels or avoiding intestinal infarction, embolization of main trunk arteries may be safe due to the development of collateral arteries or rich communications of celiac and superior mesenteric arteries.

\section{References}

1. Buffoli F, Graffeo M, Nicosia F, Gentile C, Cesari P, Rolfi F, Paterlini A. Peptic ulcer bleeding: comparison of two hemostatic procedures. Am J Gastroenterol. 2001;96(1):89-94.

2. Encarnacion CE, Kadir S, Beam CA, Payne CS. Gastro- intestinal bleeding: treatment with gastrointestinal arterial embolization. Radiology. 1992;183(2):505-508.

3. Liou TC, Lin SC, Wang HY, Chang WH. Optimal injection volume of epinephrine for endoscopic treatment of peptic ulcer bleeding. World J Gastroenterol. 2006;12(19):31083113.

4. Lin HJ, Tseng GY, Lo WC, Lee FY, Perng CL, Chang FY, Lee SD. Predictive factors for rebleeding in patients with peptic ulcer bleeding after multipolar electrocoagulation: a retrospective analysis. J Clin Gastroenterol. 1998;26(2):113-116.

5. Loffroy R, Estivalet L, Cherblanc V, Sottier D, Guiu B, Cercueil JP, Krause D. Transcatheter embolization as the new reference standard for endoscopically unmanageable upper gastrointestinal bleeding. World J Gastrointest Surg. 2012;4(10):223-227.

6. Kim JH, Shin JH, Yoon HK, Chae EY, Myung SJ, Ko GY, Gwon DI, et al. Angiographically negative acute arterial upper and lower gastrointestinal bleeding: incidence, predictive factors, and clinical outcomes. Korean J Radiol. 2009;10(4):384-390.

7. Johnston C, Tuite D, Pritchard R, Reynolds J, McEniff N, Ryan JM. Use of provocative angiography to localize site in recurrent gastrointestinal bleeding. Cardiovasc Intervent Radiol. 2007;30(5):1042-1046.

8. Ryan JM, Key SM, Dumbleton SA, Smith TP. Nonlocalized lower gastrointestinal bleeding: provocative bleeding studies with intraarterial tPA, heparin, and tolazoline. J Vasc Interv Radiol. 2001;12(11):1273-1277.

9. Poultsides GA, Kim CJ, Orlando R, 3rd, Peros G, Hallisey MJ, Vignati PV. Angiographic embolization for gastroduodenal hemorrhage: safety, efficacy, and predictors of outcome. Arch Surg. 2008;143(5):457-461.

10. Chevallier P, Novellas S, Vanbiervliet G, Staccini P, Le Conte L, Hebuterne X, Bruneton JN. [Transcatheter embolization for endoscopically unmanageable acute nonvariceal upper gastrointestinal hemorrhage]. J Radiol. 2007;88(2):251-258.

11. Lang EK. Transcatheter embolization in management of hemorrhage from duodenal ulcer: long-term results and complications. Radiology. 1992;182(3):703-707.

12. Kwak HS, Han YM, Lee ST. The clinical outcomes of transcatheter microcoil embolization in patients with active lower gastrointestinal bleeding in the small bowel. Korean J Radiol. 2009;10(4):391-397.

13. Wong TC, Wong KT, Chiu PW, Teoh AY, Yu SC, Au KW, Lau JY. A comparison of angiographic embolization with surgery after failed endoscopic hemostasis to bleeding peptic ulcers. Gastrointest Endosc. 2011;73(5):900-908. 